\title{
Human brainnetome atlas: a new chapter of brain cartography
}

\author{
George Paxinos \\ Neuroscience Research Australia and School of Medical Sciences, the University of New South Wales Australia, Sydney, NSW 2052 Australia
}

Received July 17, 2016; accepted July 21, 2016; published online July 29, 2016

Citation: Paxinos, G. (2016). Human brainnetome atlas: a new chapter of brain cartography. Sci China Life Sci 59, 965-967. doi: 10.1007/s11427-016-5110-x

Much like travelers, brain researchers need maps to navigate the brain and map its functions. Brain atlases play a central role in neuroscience and clinical practice, and are a prerequisite for studying brain networks across scales. Brain cartography, the art and science of building brain atlases, is aimed at defining regional boundaries. For the human, a pioneering work is the widely used partial cortical map of Brodmann (Brodmann, 1909), though, paradoxically, the hardly used von Economo and Koskinas (von Economo and Koskinas, 1925) work is far better, identifying about twice as many areas. While the von Economo and Koskinas atlas has been ignored by human researcher, the non-human primate researchers have used it. In our atlas The Marmoset Brain in Stereotaxic Coordinates (Paxinos et al., 2012)(eBook is free on my web site), we used a number of the von Economo and Koskinas delineations. One of the problems of human cortical maps is that they resemble maps of Europe in different millennia.

Broadly speaking, for cortical segmentation there are four criteria: architecture, connectivity, topographic organization, and functional properties. It has become increasingly important and interesting to identify brain regions by multiple criteria. While many techniques have emerged over the last decade to map both regional specialization and distant network interactions based on each of these criteria, attempts to integrate them are sparse. Relevant to this, the recently released Brainnetome Atlas (Fan et al., 2016) is a step into a new approach to brain cartography.

The human Brainnetome Atlas is based on in vivo images, consisting of 105 cortical and 18 subcortical brain

email: g.paxinos@neura.edu.au subregions per hemisphere. The voxels within each subregion have similar whole-brain connectivity profiles derived from diffusion magnetic resonance imaging (dMRI). More importantly, each region is provided with detailed anatomical and functional connectivity profiles, as well as functional characterizations. This atlas is not only the first utilizing connectivity profiles to parcellate the entire brain, but also a paradigm shift in the field of brain cartography: from depicting cerebral anatomy and connections to constructing anatomical scaffolds on which multi-scale, multi-modal information can be organized, integrated, and investigated.

The multi-modal MRI data, including structural MRI (sMRI), dMRI and resting-state functional MRI (rs-fMRI), that were used to build the Brainnetome Atlas were obtained from 40 healthy adult volunteers who participated in the Human Connectome Project. Macroanatomic parcellations of the cortical and subcortical areas as defined in the Desikan-Killiany atlas (Desikan et al., 2006) were used as a starting point. A dedicated clustering algorithm, which had been validated in various brain areas, was then used to subdivide each area. It is difficult, if not impossible, to establish exact correspondences between the Broadmann map and the human Brainnetome Atlas, given the different criteria behind the two parcellation schemes. However, to assist other researchers in making comparisons between the two, the authors assigned tentative labels to each region, following the nomenclature in partial cortical map of Brodmann.

To decode the functions associated with each region, the authors used BrainMap (Fox and Lancaster, 2002), the largest database of its kind. The activation likelihood ratio of each region in a variety of behavioural domains and 
experimental paradigm clusters were calculated and incorporated into the atlas. This not only provides important information, but also gives a working example of organizing and integrating heterogeneous information onto an anatomical scaffold. It is expected that information, be it genetic, cognitive or behavioural, can be attached to the human Brainnetome Atlas in a similar fashion.

One of the attractive aspects of the human Brainnetome Atlas is the high-throughput nature of the underlying methodology for brain cartography. The entire brain parcellation can be accomplished from a cohort of dozens of participants in weeks, compared to the years it took to complete the work of cytoarchitectonic JuBrain (Amunts et al., 2007). Affordable high-performance computing facilties have been utilized and a homemade atlas building pipeline, Automatic Tractography-based Parcellation Pipeline (ATPP), has been made public. In the era of big science, methodological consideration of open source and high-thoughput processing is of importance. The application of high-throughput techniques to publically available datasets is an enabling methodology, leading to the atlas building being reproducible on different datasets by different researchers.

To front-end users at research institutes and hospitals, a viewer with graphical user interface is published on the Brainnetome Atlas website at http://atlas.brainnetome.org. The entire atlas can be viewed as a maximum probabilistic map in a triplanar view and region of interest definitions using the atlas as a reference can be performed. The Brainnetome Atlas Viewer was coded in MATLAB so that it can easily be implemented into commonly used brain MRI processing pipelines. In addition, this atlas can be flexibly incorporated into common reference spaces and well-accepted software, such as the volumetric MNI space, the surface-based FreeSurfer and Caret software.

This atlas provides a new framework for human brain research and, in particular, connectome analysis that overcomes several drawbacks of previous parcellation schemes and addresses the need for a framework for integrating multi-modal information. The atlas has confirmed a number of discrepancies between earlier cytoarchitectonic maps, but has also revealed numerous anatomical subdivisions that were not previously well described.

The most important issue, before the Brainnetome Atlas is widely accepted is the validation of its delineations against cytoarchitecture. Cytoarchitecure itself is ambiguous except for the primary sensory and motor areas. If, therefore, the Brainnetome Atlas is in excellent agreement with cytoarchitectonic atlases there, it might prove to be more accurate than them elsewhere.

It would be of help too if the Brainnetome atlas preserved the macroscopically visible sulcal and gyral anatomy for intuitive description of the location, which motivated the use of the Desikan-Killiany atlas as the initial parcellation.

When the proofs of this review arrived, an article related to the human Brainnetome Atlas was published in Nature online (Glasser et al., 2016). The study of Glasser et al shares one purpose with the human Brainnetome Atlas: to parcellate the human brain into subregions. However, there are 3 notable differences between these two studies. (1) Brain coverage. The human Brainnetome Atlas covers the whole brain, including both cortical and subcortical regions, while the parcellations in Glasser et al 2016 only cover the cortical mantle. (2) Parcellation criteria. The human Brainnetome Atlas was constructed based on anatomical connectivity profiles derived from dMRI and then evaluated with rs-fMRI and BrainMap data. So it is really a whole brain atlas based on both anatomical and functional considerations. Moreover, the functions for each subregion was confirmed with BrainMap data. Although an objective semi-automated strategy was employed in Glasser et al 2016 to parcellate the human cerebral cortex using multi-modal modalities, this criterion was not used everywhere. Besides this, among the four cortical parcellation criteria (i.e. functional connectivity derived from rs-fMRI, function, architecture, and topography) to subdivide the cerebral cortex, the architecture measures (the relative cortical myelin content and cortical thickness) used in the Glasser article is worth discussing. It should be noted that the histological ways performed on the postmortem brain by traditional neuroanatomical studies are the classic methods to quantify cortical architecture. (3) Actual Boarders. There are some exact overlaps and some differences between the two maps. In the human Brainnetome Atlas, some regions of prefrontal lobe have much more fine-grained subdivisions. For example, in Broca's area, the ventral and dorsal parts of Area 44, and anterior and posterior parts of Area 45 are identified based on connectivity information. The other regions to pay attention to are the primary cortices, such as somatosensory and visual corteces. On one hand, the connectivity-based parcellation using dMRI can reveal the sensory and motor homunculi, on the other hand, the results in the human Brainnetome Atlas and other group's observations both found that different organizations in the visual areas could be mapped using different modalities. It would be interesting to systematically investigate whether a dMRI-based parcellation as in the human Brainnetome Atlas ends up matching the parcellations in Glasser et al 2016 or whether it reveals something different. Concluding the comparison, after over a century of only having the partial cortical map of Broadmann, two major cortical maps appeared within months of each other.

In summary, the human Brainnetome Atlas provides a cross-validated, group-level parcellation scheme of the human brain. The authors correctly emphasize that ultimately individual, subject-level parcellations will be required to reflect inter-individual variability in the location 
of brain modules. Thus, the Brainnetome Atlas should be regarded as an important step for creating more fine-grained atlases and it opens a new avenue for understanding brain function and dysfunction.

Compliance and ethics The author(s) declare that they have no conflict of interest.

Amunts, K., Schleicher, A., and Zilles, K. (2007). Cytoarchitecture of the cerebral cortex-More than localization. Neuroimage 37, 1061-1065.

Brodmann, K. (1909). Vergleichende Lokalisationslehre der Grosshirnrinde in ihren Prinzipien dargestellt auf Grund des Zellenbaues. Barth.

Desikan, R.S., Segonne, F., Fischl, B., Quinn, B.T., Dickerson, B.C., Blacker, D., Buckner, R.L., Dale, A.M., Maguire, R.P., Hyman, B.T.,
Albert, M.S., and Killiany, R.J. (2006). An automated labeling system for subdividing the human cerebral cortex on MRI scans into gyral based regions of interest. Neuroimage 31, 968-980.

Fan, L., Li, H., Zhuo, J., Zhang, Y., Wang, J., Chen, L., Yang, Z., Chu, C., Xie, S., Laird, A.R., Fox, P.T., Eickhoff, S.B., Yu, C., and Jiang, T. (2016). The human brainnetome atlas: a new brain atlas based on connectional architecture. Cereb Cortex 31, 3508-3526.

Fox, P.T., and Lancaster, J.L. (2002). Opinion: mapping context and content: the BrainMap model. Nat Rev Neurosci 3, 319-321.

Glasser, M.F., Coalson, T.S., Robinson, E.C., Hacker, C.D., Harwell, J., Yacoub, E., Ugurbil, K., Andersson, J., Beckmann, C.F., Jenkinson, M., Smith, S.M., and Van Essen, D.C. (2016). A multi-modal parcellation of human cerebral cortex. Nature, doi:10.1038/nature18933.

Paxinos, G., Watson, C., Petrides, M., Rosa, M., and Tokuno, H. (2012). The marmoset brain in stereotaxic coordinates. Elsevier.

von Economo, C.F., and Koskinas, G.N. (1925). Die cytoarchitektonik der hirnrinde des erwachsenen menschen. J Springer.

Open Access This article is distributed under the terms of the Creative Commons Attribution License which permits any use, distribution, and reproduction in any medium, provided the original author(s) and source are credited. 